\title{
Choosing $\mathbf{k}$ from $\mathbf{m}$ : feasible elimination procedures reconsidered
}

Citation for published version (APA):

Peleg, B., \& Peters, H. J. M. (2014). Choosing k from m: feasible elimination procedures reconsidered. Maastricht University, Graduate School of Business and Economics. GSBE Research Memoranda No. $033 \mathrm{https}: / /$ doi.org/10.26481/umagsb.2014033

Document status and date:

Published: 01/01/2014

DOI:

10.26481/umagsb.2014033

Document Version:

Publisher's PDF, also known as Version of record

\section{Please check the document version of this publication:}

- A submitted manuscript is the version of the article upon submission and before peer-review. There can be important differences between the submitted version and the official published version of record.

People interested in the research are advised to contact the author for the final version of the publication, or visit the DOI to the publisher's website.

- The final author version and the galley proof are versions of the publication after peer review.

- The final published version features the final layout of the paper including the volume, issue and page numbers.

Link to publication

\footnotetext{
General rights rights.

- You may freely distribute the URL identifying the publication in the public portal. please follow below link for the End User Agreement:

www.umlib.nl/taverne-license

Take down policy

If you believe that this document breaches copyright please contact us at:

repository@maastrichtuniversity.nl

providing details and we will investigate your claim.
}

Copyright and moral rights for the publications made accessible in the public portal are retained by the authors and/or other copyright owners and it is a condition of accessing publications that users recognise and abide by the legal requirements associated with these

- Users may download and print one copy of any publication from the public portal for the purpose of private study or research.

- You may not further distribute the material or use it for any profit-making activity or commercial gain

If the publication is distributed under the terms of Article $25 \mathrm{fa}$ of the Dutch Copyright Act, indicated by the "Taverne" license above, 


\section{Maastricht University}

Bezalel Peleg, Hans Peters

Choosing $k$ from m: feasible elimination procedures reconsidered

RM/14/033

\section{GSBE}

Maastricht University School of Business and Economics

Graduate School of Business and Economics

P.O Box 616

NL- 6200 MD Maastricht

The Netherlands 


\title{
Choosing $k$ from $m$ : feasible elimination procedures reconsidered*
}

\author{
Bezalel Peleg ${ }^{\dagger} \quad$ Hans Peters ${ }^{\ddagger}$
}

This version, September 2014

\begin{abstract}
We show that feasible elimination procedures (Peleg, 1978) can be used to select $k$ from $m$ alternatives. An important advantage of this method is the core property: no coalition can guarantee an outcome that is preferred by all its members. We also provide an axiomatic characterization for the case $k=1$, using the conditions of anonymity, Maskin monotonicity, and independent blocking. Finally, we show for any $k$ that outcomes of feasible elimination procedures can be computed in polynomial time, by showing that the problem is computationally equivalent to finding a maximal matching in a bipartite graph.
\end{abstract}

Journal of Economic Literature Classification Nos. C70, D71

Keywords Feasible elimination procedure, choosing $k$ from $m$, axiomatization, computation

\section{Introduction}

The Gibbard-Satterthwaite Theorem (Gibbard, 1973, and Satterthwaite, 1975) says that for every non-dictatorial social choice function whose range contains at least three alternatives, sincere voting is not a dominant strategy. Closely related to this, we obtain that for every non-dictatorial social choice function there exists a situation where either there exists no Nash equilibrium or the sincere outcome is not the unique Nash equilibrium outcome (see Theorem 2.1).Thus, one of the problems that a designer of voting schemes faces is strategic distortion of the outcome. This has led one of the authors (Peleg, 1978) to introduce the class of so-called exactly and strongly consistent social choice functions, which

\footnotetext{
*Financial support from GSBE, Maastricht University, is gratefully acknowledged.

${ }^{\dagger}$ Center for the Study of Rationality, The Hebrew University of Jerusalem, Jerusalem 91904, Israel. Email: pelegba@math.huji.ac.il.

${ }^{\ddagger}$ Department of Quantitative Economics, Maastricht University, Maastricht, The Netherlands. Email: h.peters@maastrichtuniversity.nl.
} 
resist distortion to a large extent. Indeed, for an exactly and strongly consistent social choice function the sincere outcome is always an outcome of a strong Nash equilibrium of the associated voting game. Of course, the sincere outcome may not be the unique outcome of a strong Nash equilibrium; however, it will always belong to the core of the relevant voting game. The foregoing paper has been followed by several investigations of the set of exactly and strongly consistent social choice functions: Dutta and Pattanaik (1978), Polishchuk (1978), Ishikawa and Nakamura (1980), Oren (1981), Kim and Roush (1981), Holzman (1986), and Peleg and Peters (2006). Also the books of Peleg (1984), Abdou and Keiding (1991), and Peleg and Peters (2010) have chapters devoted to consistent voting.

The central tool, introduced in Peleg (1978), to obtain exactly and strongly consistent social choice functions is the concept of a feasible elimination procedure. In such a procedure, applied to a profile of preferences, alternatives are eliminated one by one, until a final alternative remains: this is called a maximal alternative. In this paper we use this procedure to select not just one, but also $k>1$ alternatives, simply by taking the last $k$ instead of only the last alternative. We show that for at least two extensions of voters' preferences over the alternatives to (ordered) $k$-tuples of alternatives, this method has the core property: no coalition can guarantee an outcome ( $k$-tuple) that is preferred by all its members. Formally, this core is defined as the core of the effectivity function induced by this method. We show, by an example, that some well-known existing methods (single transferable vote, plurality, plurality with run-off) violate the core property.

We also provide an axiomatic characterization of the social choice correspondence which assigns the maximal alternatives to each profile, using the conditions of anonymity, Maskin monotonicity, and independent blocking.

Finally, we show that the problem of determining whether a specific $k$-tuple can result from a feasible elimination procedure is computationally equivalent to the problem of finding a maximal matching in a bipartite graph. The latter problem can be solved in polynomial time (Hopkroft and Karp, 1973).

Section 2 presents basic definitions and preliminary results, and Section 3 the axiomatic characterization of the social choice correspondence assigning maximal alternatives. In Section 4 we consider the extension to choosing $k$ from $m$, and in Section 5 we show that this method is polynomial. Section 6 concludes.

Notations The following basic notations are used throughout. For a set $D,|D|$ denotes the cardinality of $D, P(D)$ the power set, i.e., the set of all subsets of $D$, and $P_{0}(D)$ the set of all nonempty subsets of $D$.

\section{Preliminaries}

Let $A$ be a set of $m$ alternatives, $m \geq 2$, and let $N=\{1, \ldots, n\}, n \geq 2$, be a set of voters. Denote by $L$ the set of all linear orderings ${ }^{1}$ of $A$. A social

\footnotetext{
${ }^{1}$ I.e., complete, antisymmetric and transitive binary relations.
} 
choice function (SCF) is a function $F: L^{N} \rightarrow A$. If $R^{N} \in L^{N}$ is a profile of preferences of the voters and $F$ is an SCF, then the pair $\left(F, R^{N}\right)$ defines an ordinal $n$-person game in strategic form, in which each player (voter) has strategy set $L, F$ determines the outcome (alternative), and this outcome is evaluated by $R^{i}$ for each player $i \in N$. As usual, the profile $Q^{N} \in L^{N}$ is a Nash equilibrium (NE) of the game $\left(F, R^{N}\right)$ if $F\left(Q^{N}\right) R^{i} F\left(P^{i}, Q^{N \backslash\{i\}}\right)$ for all $i \in N$ and $P^{i} \in L$. An SCF $F$ is nonmanipulable (or strategy-proof) if for all $R^{N} \in L^{N}, R^{N}$ itself is a Nash equilibrium of the game $\left(F, R^{N}\right)$. An SCF $F$ is dictatorial if there exists a voter $d \in N$, a dictator, such that $F\left(R^{N}\right) R^{d} x$ for all $R^{N} \in L^{N}$ and all $x$ in the range of $F$. The Gibbard-Satterthwaite Theorem (Gibbard, 1973; Satterthwaite, 1975) states that if an SCF is nonmanipulable and its range contains at least three alternatives, then it is dictatorial. Thus, if an SCF $F$ is non-dictatorial and surjective and $m \geq 3$, then $F$ is manipulable; that is, there exists a preference profile $R^{N}$ that is not an NE of the game $\left(F, R^{N}\right)$.

A social choice correspondence (SCC) is a function $H: L^{N} \rightarrow P_{0}(A)$. We do not distinguish between the SCF $F$ and the SCC $H_{F}$, where $H_{F}\left(R^{N}\right)=$ $\left\{F\left(R^{N}\right)\right\}$ for all $R^{N} \in L^{N}$. An SCC $H$ is Maskin monotonic (Maskin, 1999) if it satisfies the following. Let $R^{N}, Q^{N} \in L^{N}$ and let $x \in H\left(Q^{N}\right)$. If $x Q^{i} y$ implies $x R^{i} y$ for all $y \in A$ and $i \in N$, then $x \in H\left(R^{N}\right)$.

Let $F$ be an SCF. For $R^{N} \in L^{N}$ denote

$$
N E\left(R^{N}\right)=\left\{Q^{N} \in L^{N}: Q^{N} \text { is an NE of }\left(F, R^{N}\right)\right\} .
$$

We say that $F$ is distorted if for some $R^{N} \in L^{N},\left\{F\left(R^{N}\right)\right\} \neq F\left(N E\left(R^{N}\right)\right)$. Requiring that $F$ is not distorted seems a weakening of nonmanipulability. However, we have:

Theorem 2.1. If an SCF $F$ is not distorted and its range contains at least three alternatives, then it is dictatorial.

Proof. Suppose $F$ is not distorted. Then it implements itself by Nash equilibria. Hence it is Maskin monotonic (see, e.g., Peleg, 1984, Lemma 6.5.1). Thus, by Muller and Satterthwaite (1977) $F$ is dictatorial.

An SCC $H$ is Paretian if for all $x, y \in A$ and $R^{N} \in L^{N}$, if $x \neq y$ and $y R^{i} x$ for all $i \in N$, then $x \notin H\left(R^{N}\right)$. A family of SCFs that are non-dictatorial and Paretian and 'not easily distorted' was suggested in Peleg (1978). First we need a few definitions.

Definition 2.2. Let $F$ be an SCF and let $R^{N} \in L^{N}$. A preference profile $Q^{N}$ is a strong Nash equilibrium (SNE) of $\left(F, R^{N}\right)$ if for every non-empty subset $S$ of $N$ and for every $P^{S} \in L^{S}$ there exists $i \in S$ such that $F\left(Q^{N}\right) R^{i} F\left(Q^{N \backslash S}, P^{S}\right)$. $\|$

Definition 2.3. A surjective SCF F is exactly and strongly consistent (ESC) if for every $R^{N} \in L^{N}$ there exists an $\operatorname{SNE} Q^{N}$ of $\left(F, R^{N}\right)$ such that $F\left(Q^{N}\right)=$ $F\left(R^{N}\right)$. 
An SCC $H$ is anonymous if for all $R^{N} \in L^{N}$ and for all permutations $\pi$ of $N, H\left(R^{1}, \ldots, R^{n}\right)=H\left(R^{\pi(1)}, \ldots, R^{\pi(n)}\right)$. Peleg (1978) proposed the following method, leading to an important class of anonymous ESC SCFs.

Definition 2.4. Assume that $n+1 \geq m$ and let $\beta: A \rightarrow N$ satisfy $\sum_{x \in A} \beta(x)=$ $n+1$. Let $R^{N} \in L^{N}$. A feasible elimination procedure (f.e.p.) for $R^{N}$ is a sequence $\left(x_{1}, C_{1} ; \ldots ; x_{m-1}, C_{m-1} ; x_{m}\right)$ such that

1) $C_{1}, \ldots, C_{m-1}$ are pairwise disjoint subsets of $N$ and $\left|C_{j}\right|=\beta\left(x_{j}\right)$ for $j=1, \ldots, m-1$,

2) $A=\left\{x_{1}, \ldots, x_{m}\right\}$,

3) $x_{k} R^{i} x_{j}$ for $k=j+1, \ldots, m$, all $i \in C_{j}$, and $j=1, \ldots, m-1$. $\|$

It is not difficult to see that there exists always at least one f.e.p. under the assumptions in the definition. Henceforth in this paper we assume $n+1 \geq m$. An alternative $y$ is $R^{N}$ - maximal if there exists an f.e.p. $\left(x_{1}, C_{1} ; \ldots ; x_{m-1}, C_{m-1} ; y\right)$. We denote

$$
M\left(R^{N}\right)=\left\{x \in A: x \text { is } R^{N} \text {-maximal }\right\} .
$$

$M$ is an anonymous and Paretian social choice correspondence. ${ }^{2}$ It may also be shown that it is Maskin monotonic (see, e.g., Peleg and Peters, 2010, Theorem 9.3.6; or Lemma 5.2 below). Hence, it admits an anonymous, Paretian, and monotonic selection. (An SCF $F$ is monotonic if it satisfies the following condition: if $R^{N} \in L^{N}, x=F\left(Q^{N}\right)$, and $R^{N}$ is obtained from $Q^{N}$ by improving the position of $x$ and leaving the relative positions of all other alternatives intact, then $x=F\left(R^{N}\right)$.) For instance, one may select from $M\left(R^{N}\right)$ according to a given fixed ordering in $L$. This is important in view of the following result (see, e.g., Peleg and Peters, 2010, Theorem 9.2.6).

Theorem 2.5. Every selection from $M$ is ESC.

In order to formulate the converse to Theorem 2.5 we need the following definition.

Definition 2.6. A function $E: P(N) \rightarrow P\left(P_{0}(A)\right)$ is an effectivity function (EF) if: i) $E(N)=P_{0}(A)$, ii) $A \in E(S)$ for every $S \in P_{0}(N)$, and iii) $E(\emptyset)=\emptyset$. $\|$

With a map $\beta$ as in Definition 2.4 we associate the EF $E_{\beta}$ by defining

$$
B \in E_{\beta}(S): \Leftrightarrow|S| \geq \sum_{x \in A \backslash B} \beta(x) \text { for all } B \in P_{0}(A) \text { and } S \in P_{0}(N) .
$$

An EF is anonymous if $E(S)$ depends only on $|S|$ for all $S$. Clearly, $E_{\beta}$ is anonymous. An EF may be considered as the coalition function a la von Neumann and Morgenstern of some game form. In particular, if $F$ is a surjective SCF then its EF $E^{F}$ is defined by

$$
B \in E^{F}(S): \Leftrightarrow \exists R^{S} \in L^{S}\left[F\left(R^{S}, Q^{N \backslash S}\right) \in B \text { for all } Q^{N \backslash S} \in L^{N \backslash S}\right] .
$$

\footnotetext{
${ }^{2}$ Clearly, $M$ depends on $\beta$, but this is suppressed from notation if no confusion is likely.
} 
We now have the following converse of Theorem 2.5 (see Corollary 9.3.4 and Theorem 9.3.5 in Peleg and Peters, 2010).

Theorem 2.7. An SCF $F$ is a selection from $M$ iff $F$ is ESC and $E^{F}=E_{\beta}$.

A voter $i$ is a (weak) vetoer with respect to an $\mathrm{EF} E$ if $E(i) \neq\{A\}$. It can be shown (see Example 10.5.4 in Peleg and Peters, 2010) that if an SCF $F$ is ESC and has an anonymous EF $E^{F}$ without vetoers, then there is a $\beta$ (with $\beta(x) \geq 2$ for all $x \in A$ ) such that $E^{F}=E_{\beta}$. Thus, by varying $\beta$ with this additional property, all ESC SCF's with anonymous EF and without vetoers are obtained in Theorem 2.7. In other words, if there are no vetoers, then every ESC social choice function is based on feasible elimination procedures.

\section{An axiomatic characterization of $M$}

We shall give in this section an axiomatic characterization of the SCC $M$. First, we need some new concepts. Let $H: L^{N} \rightarrow P_{0}(A)$ be an SCC. Then $\mathrm{H}$ is not imposed if for every $x \in A$ there exists $R^{N} \in L^{N}$ such that $H\left(R^{N}\right)=\{x\}$. If $H$ is not imposed then the effectivity function of $H, E^{H}$, is defined as follows. Let $S \in P_{0}(N)$ and let $B \in P_{0}(A)$. Then $B \in E^{H}(S)$ if there exists an $S$-profile $R^{S}$ such that $H\left(R^{S}, Q^{N \backslash S}\right) \subseteq B$ for all $N \backslash S$-profiles $Q^{N \backslash S}$. Further, $E^{H}(\emptyset)=\emptyset$.

We also need the following definition. Let $E: P(N) \rightarrow P\left(P_{0}(A)\right)$ be an EF, let $R^{N} \in L^{N}$, let $S \in P_{0}(N)$, let $B \in E(S)$, and let $x \in A \backslash B$. We say that $x$ is dominated by $B$ via $S$ at $R^{N}$ if $y R^{i} x$ for all $y \in B$ and $i \in S ; x$ is dominated at $R^{N}$ if there exist $B$ and $S$ as above such that $B$ dominates $x$ via $S$ at $R^{N}$. The core of $\mathrm{E}$ at $R^{N}, C\left(E, R^{N}\right)$, is the set of all alternatives that are not dominated at $R^{N}$.

For an SCC $H$ we define the function $E_{*}^{H}: P(N) \rightarrow P\left(P_{0}(A)\right)$ as follows. Let $S \in P_{0}(N)$ and $B \in P_{0}(A)$. Then $B \in E_{*}^{H}(S)$ if for all $R^{N} \in L^{N}$ it holds that $x R^{i} y$ for all $x \in B, y \in A \backslash B$ and $i \in S$ implies $H\left(R^{N}\right) \subseteq B$. Furthermore, $E_{*}^{H}(\emptyset)=\emptyset .^{3}$ Now let $B \in P_{0}(A), B \neq A$. The blocking coefficient of $B$ is defined as follows. If there exists an $S \in P_{0}(N)$ with $A \backslash B \in E_{*}^{H}(S)$, then

$$
b(B)=\min \left\{|S|: A \backslash B \in E_{*}^{H}(S)\right\} .
$$

Otherwise, we define $b(B)=n+1$. We also define $b(\emptyset)=0$. Note that $b(A)=$ $n+1$. Clearly, these blocking coefficients are well-defined for any SSC $H$; a blocking coefficient $b(B)$ is the minimum size of a coalition that can make sure that the outcomes under $H$ are in the complement $A \backslash B$ by reporting preferences where $B$ is the bottom part.

We call $H$ independently blocking if $b(\cdot)$ is additive; that is, if $B_{1}$ and $B_{2}$ are disjoint subsets of $A$ and $B$ is the union of $B_{1}$ and $B_{2}$, then $b(B)=b\left(B_{1}\right)+b\left(B_{2}\right)$. Note that, if $H$ is independently blocking, then $b(B)=n+1 \Leftrightarrow B=A$.

We can now state our characterization result.

\footnotetext{
${ }^{3}$ The function $E_{*}^{H}$ is called the first effectivity function of $H$ (Peleg, 1984). Note that $E_{*}^{H}$ is indeed an effectivity function if i) of Definition 2.6, is satisfied. This is for instance the case if $H$ is Paretian.
} 
Theorem 3.1. Let $H: L^{N} \rightarrow P_{0}(A)$ be an SCC. The following two statements are equivalent.

1) $H$ is anonymous, Maskin monotonic, and independently blocking with blocking coefficients $\beta(x), x \in A$.

2) $H$ coincides with the $S C C M$, determined by feasible elimination procedures with respect to the blocking coefficients $\beta(x), x \in A$.

Proof. For the implication 2) $\Rightarrow 1$ ), let $M$ be determined by blocking coefficients $\beta(x), x \in A$. We have already mentioned that $M$ is anonymous and Maskin monotonic. Let $b(B), B \in P(A)$, be the blocking coefficients associated with $E_{*}^{M}$. To show that $M$ is independently blocking it is sufficient to show that $b(B)=\beta(B)$ for all $B \in P_{0}(A), B \neq A{ }^{4}$

First, let $B \in P_{0}(A)$ with $B \neq A$ and let $S \in P(N)$ with $|S|=\beta(B)$. Let $R^{N} \in L^{N}$ with $(A \backslash B) R^{S} B$, and suppose $x \in M\left(R^{N}\right) \cap B$. Let $\left(x_{1}, C_{1} ; \ldots\right.$; $\left.x_{m-1}, C_{m-1} ; x\right)$ be an f.e.p. resulting in $x$. Since $x \in B$ and therefore $S \cap C_{k}=\emptyset$ for all $x_{k} \in A \backslash B$, we have $C_{k} \subseteq N \backslash S$ for all $x_{k} \in A \backslash B$, so that $|N \backslash S| \geq \beta(A \backslash B)$. Thus, $n=|N \backslash S|+|S| \geq \beta(A \backslash B)+\beta(B)=n+1$, a contradiction. Therefore, $M\left(R^{N}\right) \subseteq A \backslash B$, and hence $A \backslash B \in E_{*}^{M}(S)$, so that $b(B) \leq|S|=\beta(B)$.

Suppose, next, that $|S|=b(B)<\beta(B)$ for some $S \in P(N)$ and $B \in P_{0}(A)$, $B \neq A$. By anonymity, this implies that $M\left(R^{N}\right) \subseteq A \backslash B$ for all $R^{N} \in L^{N}$ with $(A \backslash B) R^{S} B$. Since, however, we now have $|N \backslash S|=n-|S|>n-\beta(B)=$ $\beta(A \backslash B)-1$, hence $|N \backslash S| \geq \beta(A \backslash B)$, it is easy to find a profile $R^{N \backslash S}$ for $N \backslash S$ for which there is an f.e.p. eliminating all alternatives of $A \backslash B$. This contradicts the fact that $A \backslash B \in E_{*}^{M}(S)$. From this contradiction we conclude that $b(B) \geq \beta(B)$.

Thus, $b(B)=\beta(B)$ for all $B \in P_{0}(A)$, and the proof of the implication $2) \Rightarrow 1)$ is complete.

We now prove the implication 1) $\Rightarrow 2$ ). So let $H$ be anonymous, Maskin monotonic, and independently blocking with coefficients $\beta(x), x \in A$. Then for these blocking coefficients $\beta(x), M$ is well defined.

Let $R^{N} \in L^{N}$. We first prove that $M\left(R^{N}\right) \subseteq H\left(R^{N}\right)$. Let $x \in M\left(R^{N}\right)$. Then there exists an f.e.p. $\left(x_{1}, C_{1} ; \ldots ; x_{m-1}, C_{m-1} ; x\right)$ with respect to $R^{N}$. Let now $Q^{N}$ be the profile that is obtained from $R^{N}$ by lowering $x_{j}$ to the bottom of $R^{i}$ for all $i \in C_{j}$ and for $j=1, \ldots, m-1$, and leaving everything else intact. By the definition of blocking coefficients, $H\left(Q^{N}\right) \subseteq A \backslash\left\{x_{j}\right\}$ for all $j=1, \ldots, m-1$, so that $H\left(Q^{N}\right)=\{x\}$. Finally, since $x R^{i} x_{j}$ for all $i \in C_{j}$ and $j=1, \ldots, m-1$, and since $H$ is Maskin monotonic, $x \in H\left(R^{N}\right)$.

For the reverse inclusion, let $x \in H\left(R^{N}\right)$. It is sufficient to prove that $x \in C\left(E_{\beta}, R^{N}\right)$, since $M\left(R^{N}\right)=C\left(E_{\beta}, R^{N}\right)$ by Theorem 9.3.6 in Peleg and Peters (2010). Suppose there is an $S \in P(N)$ and $B \in E_{\beta}(S)$ with $x \notin B$, such that $y R^{i} x$ for all $i \in S$ and $y \in B$. For each $i \in S$ let $Q^{i} \in L$ be a preference with $y Q^{i} z \Leftrightarrow y R^{i} z$ for all $y, z \in A \backslash B$ and with $y Q^{i} z$ for all $y \in B$ and $z \in A \backslash B$. By definition of $E_{*}^{H}, x \notin H\left(Q^{S}, R^{N \backslash S}\right)$. On the other hand, $x \in H\left(R^{N}\right)$ and

\footnotetext{
${ }^{4}$ Here and in the sequel, $\beta(B):=\sum_{x \in B} \beta(x)$.
} 
Maskin monotonicity of $H$ imply $x \in H\left(Q^{S}, R^{N \backslash S}\right)$, a contradiction. Hence, $x \in C\left(E_{\beta}, R^{N}\right)$.

We now show that the three properties in Theorem 3.1 are logically independent. It is not difficult to see that there exist anonymous selections from $M$ : e.g., take $Q \in L$ and let $F\left(R^{N}\right)$ be the alternative of $M\left(R^{N}\right)$ that is maximal according to $Q$. Such selections will be independently blocking by Theorem 2.7. However, they cannot be Maskin monotonic (if $m \geq 3$ ) because of Muller and Satterthwaite (1977) - see the proof of Theorem 2.1. Also, the Pareto correspondence is anonymous and Maskin monotonic, but it is not independently blocking: $b(B)=n$ for every $B \in P_{0}(A), B \neq A$. For the independence of anonymity we consider the following example.

Example 3.2. Let $A=\{x, y\}$ and let $N=\{1,2,3,4\}$. Define an EF $E$ by the following rules. Let $E(\emptyset)=\emptyset ; E(S)=\{\{y\}, A\}$ if $S=\{2,3,4\} ; E(S)=$ $\{\{x\}, A\}$ if $S \in\{\{1,2\},\{1,2,3\},\{1,2,4\}\} ; E(N)=P_{0}(A)$; and $E(S)=\{A\}$ otherwise. Let further $H\left(R^{N}\right)=C\left(E, R^{N}\right)$ for all $R^{N} \in L^{N}$. Then $H$ is Maskin monotonic, and independently blocking: $b(x)+b(y)=3+2=5=b(A)$. However, $H$ is not anonymous. $\|$

\section{Choosing $k$ from $m$}

In this section we show how the concept of a feasible elimination procedure can be used to select not just one alternative, but an ordered $k$-tuple of alternatives - where $1 \leq k \leq m-1$. We show, in particular, that the resulting method has the core property: there is no subset of voters that can vote strategically in order to guarantee a better outcome for all its members. This is in contrast to some well-known existing methods that can be used for choosing $k$ from $m-$ see Example 4.4.

Formally, let $k \in\{1, \ldots, m\}$ and denote by $\bar{A}^{k}$ the set

$$
\left\{\left(x_{1}, \ldots, x_{k}\right) \in A^{k}:\left|\left\{x_{1}, \ldots, x_{k}\right\}\right|=k\right\} .
$$

As before, assume that $n+1 \geq m$ and let $\beta: A \rightarrow N$ satisfy $\sum_{x \in A} \beta(x)=n+1$. For these weights, we define $M^{k}: L^{N} \rightarrow P_{0}\left(\bar{A}^{k}\right)$ by: $\left(y_{1}, \ldots, y_{k}\right) \in M^{k}\left(R^{N}\right)$ if there is an f.e.p. $\left(x_{1}, C_{1} ; \ldots ; x_{m-1}, C_{m-1} ; x_{m}\right)$ such that $\left(y_{1}, \ldots, y_{k}\right)=\left(x_{m-k+1}\right.$, $\left.\ldots, x_{m}\right)$. In words, $M^{k}$ assigns to a preference profile all tuples of $k$ last alternatives in any f.e.p. for that profile. Clearly, $M^{1}=M$. Define the function $E^{M^{k}}: P(N) \rightarrow P\left(P_{0}\left(\bar{A}^{k}\right)\right)$ by $E^{M^{k}}(\emptyset)=\emptyset$ and, for $S \neq \emptyset$ and $B^{k} \in \bar{A}^{k}$ :

$$
B^{k} \in E^{M^{k}}(S): \Leftrightarrow \exists R^{S} \in L^{S}\left[\forall R^{N \backslash S} \in L^{N \backslash S}: M^{k}\left(R^{S}, R^{N \backslash S}\right) \subseteq B^{k}\right] .
$$

Since, in particular, $\left\{\left(x_{1}, \ldots, x_{k}\right)\right\} \in E^{M^{k}}(N)$ for each $\left(x_{1}, \ldots, x_{k}\right) \in \bar{A}^{k}$ (for each $i \in N$ let $R^{i}$ satisfy $\left.x_{k} R^{i} x_{k-1} \ldots x_{2} R^{i} x_{1} R^{i} \ldots\right)$, we have that $E^{M^{k}}$ is an effectivity function: it is the effectivity function associated with $M^{k}$.

In this paper we consider two kinds of preferences of the voters for $k$-tuples. Both kinds of preferences extend the original preferences in $L$ to linear orderings 
on $\bar{A}^{k}$. In the first extension we respect the order of a $k$-tuple and lexicographically compare alternatives, starting from the last one. Formally we have:

(P1) Let $R \in L$. Then for $\bar{x}=\left(x_{1}, \ldots, x_{k}\right) \in \bar{A}^{k}$ and $\bar{y}=\left(y_{1}, \ldots, y_{k}\right) \in \bar{A}^{k}$ we define $\bar{x} \bar{R} \bar{y}$ if $x_{\ell} R y_{\ell}$, where $\ell=\max \left\{j \in\{1, \ldots, k\}: x_{j} \neq y_{j}\right\}$.

In the second extension we ignore the order of a $k$-tuple and lexicographically compare alternatives, starting from the worst one. Formally we have:

(P2) Let $R \in L$. For $\bar{x}=\left(x_{1}, \ldots, x_{k}\right) \in \bar{A}^{k}$ and $\bar{y}=\left(y_{1}, \ldots, y_{k}\right) \in \bar{A}^{k}$ reorder the alternatives so that $x_{i_{1}} R \ldots R x_{i_{k}}$ and $y_{j_{1}} R \ldots R y_{j_{k}}$. Then we define $\bar{x} \bar{R} \bar{y}$ if $x_{i_{\ell}} R y_{j_{\ell}}$, where $\ell=\max \left\{h \in\{1, \ldots, k\}: x_{i_{h}} \neq y_{j_{h}}\right\}$.

Preference extension P1 could apply, for instance, if a president and a vicepresident of a society have to be chosen $(k=2)$. Then we first compare the candidates for president and, in case these are equal, we compare the candidates for vice-president. Extension P2 could apply when the members of a board to be chosen are on equal foot. Then we first compare the worst candidates. If these are the same, then we compare second worst candidates, etc. ${ }^{5}$

Let $E^{k}: P(N) \rightarrow P\left(P_{0}\left(\bar{A}^{k}\right)\right)$ be an effectivity function and let $\bar{R}^{N}$ be a profile of linear orderings on $\bar{A}^{k}$. Then the core $C\left(E^{k}, \bar{R}^{N}\right)$ is defined in the usual way. We will now show that $M^{k}$ selects only core alternatives under both preference extension (P1) and preference extension (P2), starting with the latter.

Theorem 4.1. Let $R^{N} \in L^{N}$, and let $\bar{R}^{N}$ be the profile of extended preferences according to (P2). Then $M^{k}\left(R^{N}\right) \subseteq C\left(E^{M^{k}}, \bar{R}^{N}\right)$.

Proof. Consider an f.e.p. $f^{*}=\left(x_{1}, C_{1} ; \ldots ; x_{m-1}, C_{m-1} ; x_{m}\right)$ for $R^{N}$, resulting in $\left(x_{m-k+1}, \ldots, x_{m}\right) \in M^{k}\left(R^{N}\right)$. Suppose there is an $S \subseteq N$ and a set $B^{k} \subseteq \bar{A}^{k}$ such that $B^{k} \in E^{M^{k}}(S),\left(x_{m-k+1}, \ldots, x_{m}\right) \notin B^{k}$, and $\bar{y} \bar{R}^{i}\left(x_{m-k+1}, \ldots, x_{m}\right)$ for all $i \in S$ and $\bar{y} \in B^{k}$. We derive a contradiction, which completes the proof of the theorem.

Let $K=\left\{x_{m-k+1}, \ldots, x_{m}\right\}$ and let

$$
B=\left\{x \in A: x \in\left\{y_{1}, \ldots, y_{k}\right\} \text { for some }\left(y_{1}, \ldots, y_{k}\right) \in B^{k}\right\} .
$$

Note that $B \backslash K \neq \emptyset$, otherwise we would have $B=K$, which is not possible in view of the preferences of the voters in $S$. Consider any $x_{j} \in B \backslash K$, and $\bar{y}=\left(y_{1}, \ldots, y_{k}\right) \in B^{k}$ with $y_{\ell}=x_{j}$ for some $\ell \in\{1, \ldots, k\}$. Since $\bar{y} \bar{R}^{i}\left(x_{m-k+1}, \ldots, x_{m}\right)$ for all $i \in S$, by (P2) there is for each $i \in S$ an $x_{h} \in K$ with $x_{j} R^{i} x_{h}$. Since $x_{j}$ is eliminated according to $f^{*}$ prior to the alternatives of $K$, this implies that $C_{j} \cap S=\emptyset$. Hence, the alternatives of $B \backslash K$ are eliminated according to $f^{*}$ via only voters in $N \backslash S$. Therefore we have $|N \backslash S| \geq \beta(B \backslash K)$.

Let $Q^{S} \in L^{S}$ be a profile resulting in $B^{k}$, i.e., $M^{k}\left(Q^{S}, V^{N \backslash S}\right) \subseteq B^{k}$ for all $V^{N \backslash S} \in L^{N \backslash S}$. Consider, in particular, a profile $V^{N \backslash S} \in L^{N \backslash S}$ such that each $x_{j} \in B \backslash K$ is a bottom alternative for at least $\beta\left(x_{j}\right)$ voters in $N \backslash S$ (this is

\footnotetext{
${ }^{5}$ For yet another extension, see Section 6 .
} 
possible since $|N \backslash S| \geq \beta(B \backslash K))$. Then, for this specific profile $\left(Q^{S}, V^{N \backslash S}\right)$, there is an f.e.p. in which all alternatives of $B \backslash K$ are eliminated first. This implies that the resulting $k$-tuple, say $\bar{y}$, of $B^{k}$ can only contain alternatives of $K$, contradicting $\bar{y} \bar{R}^{i}\left(x_{m-k+1}, \ldots, x_{m}\right)$ for all $i \in S$.

Theorem 4.2. Let $R^{N} \in L^{N}$, and let $\bar{R}^{N}$ be the profile of extended preferences according to (P1). Then $M^{k}\left(R^{N}\right) \subseteq C\left(E^{M^{k}}, \bar{R}^{N}\right)$.

Proof. Let $f^{*}, S, B^{k}$, and $Q^{S}$ be as in the proof of Theorem 4.1. The proof proceeds in several steps.

Step 1 Let

$$
\begin{aligned}
B(m)= & \left\{x_{j} \in A: j \in\{1, \ldots, m-1\}\right. \text { and } \\
& \left.x_{j}=y_{k} \text { for some }\left(y_{1}, \ldots, y_{k}\right) \in B^{k}\right\} .
\end{aligned}
$$

By (P1), we have $x_{j} R^{i} x_{m}$ for all $x_{j} \in B(m)$ and $i \in S$. Hence, $C_{j} \subseteq N \backslash S$ for all $j$ with $x_{j} \in B(m)$, so that $|N \backslash S| \geq \beta(B(m)$ ). Consider a profile $V^{N \backslash S}(m) \in L^{N \backslash S}$ where all $x_{j} \in B(m)$ are ranked at bottom positions for at least $\beta\left(x_{j}\right)$ voters in $N \backslash S$. Let $B^{k}(m)$ be the subset of $B^{k}$, consisting of all $k$-tuples which are obtained by f.e.p.'s for the profile $\left(Q^{S}, V^{N \backslash S}(m)\right)$ such that the alternatives of $B(m)$ are eliminated first, with $x_{j}$ before $x_{\ell}$ whenever $x_{j}, x_{\ell} \in B(m)$ and $j<\ell$; clearly, all these f.e.p.'s result in $x_{m}$, so that $y_{k}=x_{m}$ for all $\left(y_{1}, \ldots, y_{k}\right) \in B^{k}(m)$.

Step 2 Let

$$
\begin{aligned}
B(m-1)= & \left\{x_{j}: j \in\{1, \ldots, m-2\}\right. \text { and } \\
& \left.x_{j}=y_{k-1} \text { for some }\left(y_{1}, \ldots, y_{k-1}, x_{m}\right) \in B^{k}(m)\right\} .
\end{aligned}
$$

By (P1), we have $x_{j} R^{i} x_{m-1}$ for all $x_{j} \in B(m-1)$ and $i \in S$. Hence, $C_{j} \subseteq N \backslash S$ for all $j$ with $x_{j} \in B(m-1)$, so that $|N \backslash S| \geq \beta(B(m) \cup B(m-1))$. Consider a profile $V^{N \backslash S}(m-1) \in L^{N \backslash S}$ where all $x_{j} \in B(m) \cup B(m-1)$ are ranked at bottom positions for at least $\beta\left(x_{j}\right)$ voters in $N \backslash S$. Let $B^{k}(m-1)$ be the subset of $B^{k}(m)$, consisting of all $k$-tuples which are obtained by f.e.p.'s for the profile $\left(Q^{S}, V^{N \backslash S}(m-1)\right)$ such that the alternatives of $B(m) \cup B(m-1)$ are eliminated first, with $x_{j}$ before $x_{\ell}$ whenever $x_{j}, x_{\ell} \in B(m) \cup B(m-1)$ and $j<\ell$; clearly, all these f.e.p.'s have $\left(x_{m-1}, x_{m}\right)$ as last pair, so that $y_{k-1}=x_{m-1}$ and $y_{k}=x_{m}$ for all $\left(y_{1}, \ldots, y_{k}\right) \in B^{k}(m-1)$.

Step k Let

$$
\begin{aligned}
B(m-k+1)= & \left\{x_{j}: j \in\{1, \ldots, m-k\}\right. \text { and } \\
& \left.x_{j}=y_{1} \text { for some }\left(y_{1}, x_{m-k+2}, \ldots, x_{m}\right) \in B^{k}(m-k+2)\right\} .
\end{aligned}
$$

By (P1), we have $x_{j} R^{i} x_{m-k+1}$ for all $x_{j} \in B(m-k+1)$ and $i \in S$. Hence, $C_{j} \subseteq N \backslash S$ for all $j$ with $x_{j} \in B(m-k+1)$, so that $|N \backslash S| \geq \beta(B(m) \cup$ 
$\ldots \cup B(m-k+1))$. Consider a profile $V^{N \backslash S}(m-k+1) \in L^{N \backslash S}$ where all $x_{j} \in B(m) \cup \ldots \cup B(m-k+1)$ are ranked at bottom positions for at least $\beta\left(x_{j}\right)$ voters in $N \backslash S$. Let $B^{k}(m-k+1)$ be the subset of $B^{k}(m-k+2)$, consisting of all $k$-tuples which are obtained by f.e.p.'s for the profile $\left(Q^{S}, V^{N \backslash S}(m-k+1)\right)$ such that the alternatives of $B(m) \cup \ldots \cup B(m-k+1)$ are eliminated first, with $x_{j}$ before $x_{\ell}$ whenever $x_{j}, x_{\ell} \in B(m) \cup \ldots \cup B(m-k+1)$ and $j<\ell$; clearly, all these f.e.p.'s have $\left(x_{m-k+1}, \ldots, x_{m}\right)$ as last $k$-tuple. This, however, implies that $\left(x_{m-k+1}, \ldots, x_{m}\right) \in B^{k}$, which is a contradiction.

The following example shows that the converses of Theorems 4.1 and 4.2 do not hold for $k>1$. (For $k=1$ we do have $M\left(R^{N}\right)=C\left(E^{M}, R^{N}\right)$ for all $R^{N} \in L^{N}$ by Lemma 9.3.2 and Theorem 9.3.6 in Peleg and Peters, 2010.)

Example 4.3. Let $A=\{x, y, z\}, N=\{1, \ldots, 4\}, \beta(x)=1$, and $\beta(y)=\beta(z)=$ 2. Consider the profile $R^{N}$ given in the following table:

\begin{tabular}{cccc}
\hline$R^{1}$ & $R^{2}$ & $R^{3}$ & $R^{4}$ \\
\hline$y$ & $y$ & $x$ & $x$ \\
$z$ & $z$ & $z$ & $y$ \\
$x$ & $x$ & $y$ & $z$
\end{tabular}

Then $M^{2}\left(R^{N}\right)=\{(z, y)\}$. We claim that the pair $(x, y)$ is in $C\left(E^{M^{2}}, \bar{R}^{N}\right)$ according to preference assumption (P2). First, $(x, y)$ is top-ranked for voter 4. Voter 3 only prefers $(x, z)$ or $(z, x)$ to $(x, y)$ but this is not the case for voters 1 and 2. However, voter 3 alone is not effective for $\{(x, z),(z, x)\}$. Finally, voters 1 and 2 only prefer $(y, z)$ and $(z, y)$ to $(x, y)$, but $\{1,2\}$ is not effective for $\{(y, z),(z, y)\}$. We conclude that $(x, y)$ is in $C\left(E^{M^{2}}, \bar{R}^{N}\right)$ under assumption (P2).

Now consider preference assumption (P1). We claim that $(x, y)$ is still in $C\left(E^{M^{2}}, \bar{R}^{N}\right)$. Voter 4 finds $(z, x)$ and $(y, x)$ better than $(x, y)$; also voter 3 finds $(z, x)$ and $(y, x)$ better than $(x, y)$, but voters 1 and 2 prefer $(x, y)$ over $(z, x)$ and $(y, x)$, and $\{3,4\}$ is not effective for $\{(z, x),(y, x)\}$ : e.g., $\{1,2\}$ can put $x$ at bottom so that $x$ can be eliminated first. Voter 3 prefers every alternative in the set $\{(y, x),(z, x),(y, z),(x, z)\}$ to $(x, y)$, but 1 and 2 prefer only $(z, y)$ over $(x, y)$, and $\{3\}$ is not effective for $\{(y, x),(z, x),(y, z),(x, z)\}:\{1,2,4\}$ can put $x$ and $z$ at bottom so that $y$ ends up last. Finally, $\{1,2\}$ is not effective for $\{(z, y)\}$ : e.g., $\{3,4\}$ can put $z$ at bottom so that $z$ can be eliminated first. We conclude that $(x, y)$ is in $C\left(E^{M^{2}}, \bar{R}^{N}\right)$ also under assumption (P1). ॥

The fact that $M^{k}$ satisfies the core property as in Theorems 4.1 and 4.2 means that there is never a coalition of voters which can guarantee an outcome ( $k$-tuple) that is better for all its members in case $M^{k}$ is used, i.e., if a feasible elimination procedure is used. This fact is certainly not shared by well-known existing methods. The following example illustrates this. 
Example 4.4. Let $A=\{a, b, c, d\}, N=\{1,2,3\}$, and $\beta(x)=1$ for all $x \in A$. Consider the profile $R^{N}$ given in the following table:

\begin{tabular}{ccc}
\hline$R^{1}$ & $R^{2}$ & $R^{3}$ \\
\hline$a$ & $b$ & $c$ \\
$b$ & $c$ & $a$ \\
$c$ & $a$ & $b$ \\
$d$ & $d$ & $d$
\end{tabular}

Suppose we have to select two candidates. In this case, $M^{2}$ is quite inconclusive: $M^{2}\left(R^{N}\right)=\left\{(x, y) \in \bar{A}^{2}: x \neq d, y \neq d\right\}$. Still, by Theorems 4.1 and 4.2 , no coalition of voters can improve on any of the pairs in $M^{2}\left(R^{N}\right)$. Now consider the method of single transferable vote (STV - see for instance Brams and Fishburn, 2002). According to STV, first $d$ is eliminated, but thereafter either $a$ or $b$ or $c$ is eliminated. For instance, if $a$ is eliminated, then next $b$ is chosen and finally $c$, so that in our notation the pair $(c, b)$ results. This way, we obtain $S T V\left(R^{N}\right)=\{(c, b),(b, a),(a, c)\}$. Now take, for instance, the pair $(b, a)$. Both voters 2 and 3 prefer the pair $(b, c)$ over $(b, a)$ - under both (P1) and (P2) - and, moreover, under STV the coalition $\{2,3\}$ is effective for $\{(b, c)\}$ by the strategy profile

\begin{tabular}{cc}
\hline$Q^{2}$ & $Q^{3}$ \\
\hline$c$ & $c$ \\
$b$ & $b$ \\
$a$ & $a$ \\
$d$ & $d$
\end{tabular}

In fact, under STV the core for the profile in this example (which is a slightly modified version of the Condorcet paradox) is empty. It is easy to see that the same example applies to methods like plurality voting or run-off elections, assuming that in case of ties we can choose arbitrarily between tied alternatives, as in STV or feasible elimination procedures. ॥

\section{A method for computing $M^{k}$ in polynomial time}

The following lemma was proved in Peleg (1984, Lemma 5.3.5) for the case $k=1$.

Lemma 5.1. Let $R^{N} \in L^{N}$. Then $\left(x_{m-k+1}, \ldots, x_{m}\right) \in M^{k}\left(R^{N}\right)$ if and only if there exist pairwise disjoint subsets $S(y), y \in A \backslash\left\{x_{m}\right\}$, of $N$ such that

(1) $x_{j} R^{i} x_{\ell}$ for all $\ell=m-k+1, \ldots, m-1, j>\ell$, and $i \in S\left(x_{\ell}\right)$, 
(2) $x_{j} R^{i} y$ for all $y \in A \backslash\left\{x_{m-k+1}, \ldots, x_{m}\right\}, j=m-k+1, \ldots, m$, and $i \in S(y)$,

(3) $|S(y)|=\beta(y)$ for all $y \in A \backslash\left\{x_{m}\right\}$.

In the proof of this lemma we will use the fact that $M^{k}$ is Maskin monotonic. In general, a function $H: L^{N} \rightarrow P_{0}\left(\bar{A}^{k}\right)$ is Maskin monotonic if it satisfies the following. Let $R^{N}$ and $Q^{N}$ be in $L^{N}$ and let $\left(x_{1}, \ldots, x_{k}\right) \in H\left(Q^{N}\right)$. If $x_{j} Q^{i} y$ implies $x_{j} R^{i} y$ for all $j \in\{1, \ldots, k\}, y \in A$ and $i \in N$, then $\left(x_{1}, \ldots, x_{k}\right) \in$ $H\left(R^{N}\right)$. For $k=1$, this definition coincides with the definition of Maskin monotonicity in Section $2 .{ }^{6}$

Lemma 5.2. $M^{k}$ is Maskin monotonic.

Proof. Let $Q^{N}$ and $R^{N}$ as in the definition of Maskin monotonicity. Without loss of generality we assume that there is a voter $v$ such that $Q^{N \backslash\{v\}}=R^{N \backslash\{v\}}$. Let $f^{*}=\left(x_{1}, C_{1} ; \ldots ; x_{m-1}, C_{m-1} ; x_{m}\right)$ be an f.e.p. for $Q^{N}$. If $v \notin C_{1} \cup \ldots \cup C_{m-k}$ then it is easy to see that $f^{*}$ is still an f.e.p. for $R^{N}$, so that $\left(x_{m-k+1}, \ldots, x_{m}\right) \in$ $M^{k}\left(R^{N}\right)$. Now assume $v \in C_{1} \cup \ldots \cup C_{m-k}$. If $v \in C_{j}$ with $j>1$, then we may eliminate $x_{1}, \ldots, x_{j-1}$ and all voters in $C_{1} \cup \ldots \cup C_{j-1}$ first, and next continue the argument with the remaining profile, where now all voters in $C_{j}$ have $x_{j}$ bottom ranked according to $Q$. So, without loss of generality, let $v \in C_{1}$.

The rest of the proof is based on a three step algorithm.

Step 1 If the bottom alternative of $R^{v}$ is equal to $x_{1}$, then $f^{*}$ is still an f.e.p. for $\overline{R^{N}}$ and we are done. Otherwise, go to Step 2.

Step 2 Let the bottom alternative of $R^{v}$ be $x_{\ell} \neq x_{1}$, so $\ell \in\{2, \ldots, m-k\}$. If all voters in $C_{\ell}$ have $x_{\ell}$ as bottom alternative in $R^{N}$, then we can first eliminate $x_{\ell}$ via $C_{\ell}$ and go back to Step 1 for the reduced profile. Otherwise, go to Step 3.

Step 3 Take $\hat{v} \in C_{\ell}$ with $x_{\ell}$ not as bottom alternative and note that the bottom alternative of $R^{\hat{v}}=Q^{\hat{v}}$ is some $x_{j}$ with $j<\ell$ (since $x_{j}$ must be eliminated before $x_{\ell}$ in $\left.f^{*}\right)$. Then modify $C_{\ell}$ to $\hat{C}_{\ell}=\left(C_{\ell} \cup\{v\}\right) \backslash\{\hat{v}\}$ and modify $C_{1}$ to $\hat{C}_{1}=\left(C_{1} \cup\{\hat{v}\}\right) \backslash\{v\}$. (In words, we switch $v$ and $\hat{v}$.) Go back to Step 1 .

Repeat this procedure until the final substitute of $v$ in the modified $C_{1}$ has $x_{1}$ at bottom. Then we can apply an f.e.p. resulting in $\left(x_{m-k+1}, \ldots, x_{m}\right)$, so that $\left(x_{m-k+1}, \ldots, x_{m}\right) \in M^{k}\left(R^{N}\right)$.

Proof of Lemma 5.1. The only-if direction follows immediately from the definition of an f.e.p. For the if-direction, let $\left(x_{m-k+1}, \ldots, x_{m}\right) \in \bar{A}^{k}$ and assume that there exist subsets $S(y)$ of $N$, satisfying (1), (2), and (3). List the alternatives of $A \backslash\left\{x_{m-k+1}, \ldots, x_{m}\right\}$ as $\left\{x_{1}, \ldots, x_{m-k}\right\}$. Consider the following profile $Q^{N} \in L^{N}$. For $j=1, \ldots, m-k$ and $i \in S\left(x_{j}\right)$ shift $x_{j}$ to the bottom of $R^{i}$, leaving everything else intact. For all other voters $i$, let $Q^{i}=R^{i}$. Then $\left(x_{1}, S\left(x_{1}\right) ; \ldots ; x_{m-1}, S\left(x_{m-1}\right) ; x_{m}\right)$ is an f.e.p. for $Q^{N}$, so that $\left(x_{m-k+1}, \ldots, x_{m}\right)$

\footnotetext{
${ }^{6}$ Also note that this extended definition of Maskin monotonicity would be implied by Maskin monotonicity in terms of extended preferences according to (P1) or (P2) or any other sensible preference extension.
} 
$\in M^{k}\left(Q^{N}\right)$. By Maskin monotonicity of $M^{k}$ (see Lemma 5.2$),\left(x_{m-k+1}, \ldots, x_{m}\right)$ $\in M^{k}\left(R^{N}\right)$.

Lemma 5.1 can be used to determine if an alternative $\left(x_{m-k+1}, \ldots, x_{m}\right)$ of $\bar{A}^{k}$ is in $M^{k}\left(R^{N}\right)$, as follows. We define a bipartite graph with the voters of $N$ as vertices on one side, and for every $y \in A \backslash\left\{x_{m}\right\}$ we take $\beta(y)$ vertices on the other side. For every vertex corresponding to an alternative $y \in A \backslash\left\{x_{m-k+1}, \ldots, x_{m}\right\}$, we let there be an edge between this vertex and the vertex corresponding to a voter $i$ if and only if $x_{j} R^{i} y$ for every $j=m-k+1, \ldots, m$. For every vertex corresponding to an alternative $x_{\ell}$ for some $\ell \in\{m-k+1, \ldots, m\}$, we let there be an edge between this vertex and the vertex corresponding to a voter $i$ if and only if $x_{j} R^{i} x_{\ell}$ for all $j>\ell$. Then, by Lemma $5.1,\left(x_{m-k+1}, \ldots, x_{m}\right) \in M^{k}\left(R^{N}\right)$ if and only if there is a matching for this graph with the property that all vertices corresponding to alternatives in $A \backslash\left\{x_{m}\right\}$ are matched. (Note that this matching is perfect if and only if $\sum_{y \in A \backslash\left\{x_{m}\right\}} \beta(y)=n$, in which case also all voter vertices are matched.) Clearly, such a matching must be maximal and the problem of finding a maximal matching is polynomial (see Hopcroft and Karp, 1973). Repeating the foregoing procedure $m(m-1) \cdots(m-k+1)$ times is still polynomial (in $m$ and $n) .^{7}$

\section{Concluding remarks}

We have shown in this paper that the method for choosing $k$ out of $m$, based on feasible elimination procedures, has the core property for two intuitive preferences extensions (Theorems 4.1 and 4.2). Unfortunately, as the following example shows, this result does not extend to all reasonable preference extensions.

Example 6.1. Let $A=\{w, x, y, z\}, N=\{1, \ldots, 5\}, \beta(w)=\beta(x)=1$, and $\beta(y)=\beta(z)=2$. Consider the profile $R^{N}$ given in the following table:

\begin{tabular}{ccccc}
\hline$R^{1}$ & $R^{2}$ & $R^{3}$ & $R^{4}$ & $R^{5}$ \\
\hline$y$ & $y$ & $z$ & $z$ & $x$ \\
$w$ & $w$ & $w$ & $w$ & $y$ \\
$x$ & $x$ & $x$ & $x$ & $z$ \\
$z$ & $z$ & $y$ & $y$ & $w$
\end{tabular}

Then $(w, x) \in M^{2}\left(R^{N}\right)$ by the f.e.p. $(z,\{1,2\} ; y,\{3,4\} ; w,\{5\} ; x)$. We show that $(w, x) \neq C\left(E^{M^{2}}, \bar{R}^{N}\right)$ where, for each $i \in N, \bar{R}^{i}$ is the lexicographic preference extension obtained by first comparing best alternatives and then second best alternatives. Consider the following profile for the coalition $\{1, \ldots, 4\}$ :

\footnotetext{
${ }^{7}$ We thank Ilan Nehama of the Center for the Study of Rationality, Jerusalem, for helpful comments on this subject.
} 


\begin{tabular}{cccc}
\hline$Q^{1}$ & $Q^{2}$ & $Q^{3}$ & $Q^{4}$ \\
\hline$y$ & $y$ & $y$ & $y$ \\
$z$ & $z$ & $z$ & $z$ \\
$x$ & $x$ & $x$ & $x$ \\
$w$ & $w$ & $w$ & $w$
\end{tabular}

Then $M^{2}\left(Q^{\{1, \ldots, 4\}}, \widetilde{R}^{5}\right)=\{(z, y)\}$ for all $\widetilde{R}^{5} \in L$, which implies that $\{(z, y)\} \in$ $E^{M^{2}}(\{1, \ldots, 4\})$. Since $(z, y) \bar{R}^{i}(w, x)$ for all $i \in\{1, \ldots, 4\}$, we conclude that $(w, x) \notin C\left(E^{M^{2}}, \bar{R}^{N}\right) . \|$

We conclude with a few thoughts on neutrality. An SCC is neutral if it is covariant under permutations of the alternatives. To obtain neutrality of $M$ or, more generally, $M^{k}$ one needs that all weights $\beta(x)$ are equal, but this is not always possible, due to the restrictions on $\beta(\cdot)$. Of course, one can always choose the weights such that the difference between any two weights is at most one. Also, if the number of voters is large relative to the number of alternatives, then one practically obtains neutrality: e.g., if $m=10$ and $n=1000$ then one can choose nine of the weights equal to 100 and one weight equal to 101.

Alternatively, write $M_{\beta}^{k}$ if the weight function is $\beta: A \rightarrow N$. Then one could enlarge $M_{\beta}^{k}$ to $\widetilde{M}_{\beta}^{k}$ by defining

$$
\widetilde{M}_{\beta}^{k}\left(R^{N}\right)=\bigcup_{\pi \in \Pi(A)} M_{\beta \circ \pi}^{k}\left(R^{N}\right)
$$

for all $R^{N} \in L^{N}$, where $\Pi(A)$ is the set of permutations of $A$. It is not clear, however, whether this enlarged neutral SCC still has the core property.

\section{References}

Abdou J, Keiding H (1991) Effectivity Functions in Social Choice. Kluwer Academic Publishers, Dordrecht

Brams SJ, Fishburn PC (2002) Voting procedures. In: Handbook of Social Choice and Welfare, Volume 1. Elsevier, Amsterdam

Dutta B, Pattanaik PK (1978) On nicely consistent voting systems. Econometrica 46:163-170

Gibbard A (1973) Manipulation of voting schemes: A general result. Econometrica 41:587-602

Holzman R (1986) On strong representations of games by social choice functions. Journal of Mathematical Economics 15:39-57

Hopcroft JE, Karp RM (1973) An $n^{5 / 2}$ algorithm for maximum matchings in bipartite graphs. SIAM Journal on Computing 2:225-231 
Ishikawa S, Nakamura K (1980) Representations of characteristic function games by social choice functions. International Journal of Game Theory 9:191-199

Kim KH, Roush FW (1981) Properties of consistent voting systems. International Journal of Game Theory 10:45-52

Maskin E (1999) Nash equilibrium and welfare optimality. The Review of Economic Studies 66:23-38

Muller E, Satterthwaite MA (1977) The equivalence of strong positive association and strategy-proofness. Journal of Economic Theory 14:412-418

Oren I (1981) The structure of exactly strongly consistent social choice functions. Journal of Mathematical Economics 8:207-220

Peleg B (1978) Consistent voting systems. Econometrica 46:153-161

Peleg B (1984) Game Theoretic Analysis of Voting in Committees. Cambridge University Press, Cambridge

Peleg B, Peters H (2006) Consistent voting systems with a continuum of voters. Social Choice and Welfare 27:477-492

Peleg B, Peters H (2010) Strategic Social Choice. Springer, Berlin

Polishchuk I (1978) Monotonicity and uniqueness of consistent voting systems. Center for Research in Mathematical Economics and Game Theory, Hebrew University of Jerusalem

Satterthwaite MA (1975) Strategy-proofness and Arrow's conditions: Existence and correspondence theorems for voting procedures and social welfare functions. Journal of Economic Theory 10:187-207 